\title{
Imprévus et construction de la professionnalité enseignante
}

Les bouleversements du système éducatif depuis l'Ecole de Jules FERRY ont appelé à une transformation des pratiques, provoquant ainsi des modifications dans les représentations professionnelles des enseignants. Les réformes entreprises depuis la troisième République relevaient de deux "projets-visées " différents, conçus en opposition, et qui se sont retrouvés liés, par les réformes des années 80 engagées contre l'échec scolaire, dans des objectifs contradictoires - diversifier et unifier, respecter les différences et les assimiler (S. HENRIQUEZ, 1989). Et l'articulation nécessaire en a été laissée en partie aux enseignants, accroissant de la sorte l'imprévisibilité de l'enseignement, et donnant naissance à des " modèles normatifs ", conçus comme des "exigences", des lignes directrices, et repérés dans les représentations enseignantes (M. HIRSCHHORN, 1993). Les deux modèles qui nous intéressent ici de par leur valeur centrale - le savoir ou "l'enseigné " - sont ceux du magister et du pédagogue.

Nous allons présenter les résultats d'une recherche qui, souhaitant contribuer à l'étude de la professionnalité enseignante, s'est donné pour objectif de mieux connaître et de mieux comprendre les conduites des enseignants face à l'imprévu en classe. La classe, en effet, apparaît comme un lieu où des imprévus peuvent surgir, que l'enseignant doit prendre en compte s'il veut assurer la "Mission " que lui fixe la société, à travers l'Institution. L'imprévu est ainsi inscrit au cœur même de ce métier complexe (P. PERRENOUD, 1999).

Dans un premier temps nous proposerons une définition de l'imprévu et de sa gestion, puis nous indiquerons, pour une partie de notre recherche, la problématique, la méthodologie et les résultats. Nous terminerons par une interprétation des résultats et la présentation d'un outil de lecture des pratiques déclarées.

\section{UNE DEFINITION DE L'IMPREVU ET DE SA “ GESTION”}

Selon les dictionnaires (Le Grand Robert, 1985 ; QUILLET, 1986), l'imprévu serait ce qui arrive sans avoir été prévu, sans qu'on l'ait pu conjecturer, dans un contexte d'incertitude pour le sujet. Partant il surprend et déconcerte l'enseignant, activant chez lui la "fonction émotionnelle" (A-M. COSTALAT-FOUNEAU, 1997), ce qui participe de la signification du contexte par les sujets dont la conduite peut être plus ou moins bien adaptée et plus ou moins bien vécue. Nous utilisons le terme "imprévu " comme terme générique 2 , dans un sens proche de l'incident perturbateur (P. WOODS, 1990).

Le caractère "relatif" ou "radical" —arrivant à l'improviste ou inédit, "impensé" — des imprévus (P. PERRENOUD, 1999) dépend de l'expérience des enseignants confrontés à ces situations. De plus nos données montrent que pour tel enseignant, ancien dans la profession ou l'établissement, l'imprévu pourra être "relatif", alors que pour tel autre il sera " radical" : ce qui est imprévu pour l'un peut ne pas l'être pour l'autre.

Et quelle que soit la dimension concernée — contextuelle, pédagogique et didactique (N. BENAÏOUNRAMIREZ, 1999) — l'imprévu dérange, perturbe. Enseigner c'est souvent " agir dans l'urgence " et " décider dans l'incertitude ", c'est faire face à la complexité (P. PERRENOUD, 1996). Des praticiens différents, dans une même situation, n'improvisent pas de la même façon : l'imprévu agit comme un révélateur de l'habitus, selon le point de vue sociologique - et comme un révélateur des représentations, selon le point de vue psychosocial: représentations différentes en fonction de l'histoire personnelle ou professionnelle et des groupes d'appartenance professionnels et psychosociaux. Mais un même praticien n'improvisera pas non plus de la même façon dans des situations semblables à des moments différents: à la variabilité interindividuelle s'ajoute une variabilité intraindividuelle (M. BRU, 1994). Il pourrait ainsi exister chez les acteurs des divergences de conception et donc de gestion des imprévus.

\footnotetext{
${ }^{1}$ Le terme est emprunté à J. ARDOINO (2000).

${ }^{2}$ Pour plus de précisions, voir notre thèse (N. BENAÏOUN-RAMIREZ, 2001).
} 
L'adaptation à l'imprévu, selon sa nature et le contexte où il surgit, peut relever d'une improvisation bien planifiée ${ }^{3}$, de stratégies —réponse adéquate puisée dans son propre savoir d'expérience ${ }^{4}$. Elle peut relever aussi du "faire avec", de ruses, de bricolage..., car les compétences pédagogiques, à travers les pratiques relationnelles et les préoccupations éducatives, ont à gérer d'autres imprévus, relevant tout autant du “curriculum caché" que du “curriculum réel” (P. PERRENOUD, 1996).

C'est au " faire avec " que nous nous intéressons ici, dans lequel l'individu peut s'avérer selon les cas agent, acteur ou même auteur (J. ARDOINO, 2000), dans la mesure où il invente ses propres réponses.

Et si l'adaptation à l'imprévu fait partie intégrante de la mission des enseignants (B.O. du 29 mai 1997), sans que ceux-ci l'aient pour autant appris, c'est sans doute que cette circulaire a pour vocation d'inscrire la redéfinition de la mission enseignante dans une perspective.

\section{L'IMPREVU EN CLASSE DE COLLEGE : UNE RECHERCHE SUR LES REPRESENTATIONS}

\section{Une problématique psychosociale}

L'implication est une "connaissance en acte" manifestée par les acteurs dans leurs discours et leurs conduites ; elle se révèle dans les principes pas toujours conscients qui leur inspirent leurs opinions (ML. ROUQUETTE, 1997). Elle renvoie aux multi-appartenances sociales qui participent de la construction d'une identité psychosociale plurielle ${ }^{6}$ —système de sentiments et de représentations de Soi (P. TAP, 1997), fait d'altérations (J. ARDOINO, 2000), conçu comme une " unitas multiplex " (E. MORIN ${ }^{7}$ ), et orientant les conduites. L'engagement dans divers groupes produit ainsi une implication ${ }^{8}$ psychosociale qui façonne des représentations.

En contexte professionnel, les groupes professionnels —ici des enseignants de collèges publics de l'Education Nationale - se construisent des représentations professionnelles ${ }^{9}$ - ici à propos de la gestion de l'imprévu. Ces représentations orientent leurs conduites, celles-ci portant, selon C. JACQUET-MIAS (1998), la trace de leur implication professionnelle ${ }^{10}$ qui articule trois dimensions : le sens donné aux situations, les repères (valeurs) et le sentiment de contrôler la situation.

Il est donc possible d'envisager que les individus, dans la confrontation aux imprévus en situation professionnelle, produisent des réponses contextualisées, c'est-à-dire tributaires à la fois des ressources et contraintes du contexte professionnel dans lequel elles sont produites, et de la signification qu'ils donnent à ce contexte (M. BRU, 1993, 1994), signification générée par leurs représentations professionnelles, mais aussi par leur identité psychosociale. Ainsi par les réponses qu'ils apportent aux imprévus, les sujets modifient le contexte qu'ils intègrent alors en lui donnant sens, et qui modifie en retour leur conduite.

Et les implications psychosociales et professionnelles produites par les engagements psychosociaux et professionnels, à travers les représentations sociales et professionnelles, pourraient bien être considérées comme des facteurs déterminants du "faire avec" les imprévus ${ }^{11}$.

Deux des trois propositions qui ont guidé la partie empirique de la recherche font l'objet de cet article — des propositions plus que des hypothèses à "valider", car ce travail se situe dans une épistémologie de la compréhension. Elles ont porté,

- l'une sur les relations entre les variations de prises de position quant aux principes organisateurs des représentations professionnelles de la gestion de l'imprévu en classe, et leur ancrage dans les contextes et profils professionnels,

- l'autre sur les modulations de cette gestion selon l'implication professionnelle mais aussi psychosociale des enseignants.

\footnotetext{
${ }^{3}$ F-V. TOCHON (1993).

${ }^{4}$ P. PERRENOUD (1999).

${ }^{5}$ P. PERRENOUD (1999).

${ }^{6}$ Au sujet de l'identité plurielle, voir B. LAHIRE (1998).

${ }^{7}$ MORIN, E., Penser l'Europe, Gallimard, 1987.

${ }^{8}$ Au sens où, s'étant engagé, l'individu est “plié dans la situation” (M. BATAILLE, 1983 : Implication et explication. Pour, 88, 28-31.)

${ }^{9}$ Pour la définition de ces représentations sociales particulières, voir M. BATAILLE et al. (1997).

${ }^{10}$ Pour l'auteur, une manière d'être et d'investir l'espace professionnel.

11 Nous parlerons ainsi de la manière d'investir l'espace professionnel (réponses aux imprévus, implication professionnelle en trois dimensions) et de ses ancrages (engagements générant des implications psychosociales et professionnelles.
} 


\section{La démarche adoptée}

Une démarche pluriméthodologique a permis d'observer quelques-unes des multiples facettes de l'objet. Les approches qualitative et quantitative se sont relayées pour le construire, et atteindre à une certaine compréhension du "faire avec" l'imprévu en classe. Nous nous sommes ainsi intéressée aux contraintes auxquelles les enseignants se trouvent confrontés, aux significations qu'ils donnent à leurs conduites, et aux valeurs qui les orientent.

Une première étape de recherche a mis en évidence des divergences de conception des enseignants quant à l'identification des imprévus et à leur gestion (N. BENAÏOUN-RAMIREZ, 1999).

Une deuxième étape s'est attachée à repérer, par questionnaire auprès de 145 enseignants, des tendances dans ce "faire avec" les imprévus au quotidien de la classe, pour comprendre les facteurs en jeu et les processus à l'œuvre.

Le questionnaire proposé aux sujets comportait trois grandes parties :

* identité et implication psychosociales (image de Soi, engagements psychosociaux...),

* contexte et profil professionnels, implication professionnelle (engagements professionnels, travail en équipe, formation continue...), identité professionnelle (Soi et idéal professionnels),

* représentations des pratiques : planification (prévisions et déroulement de la séance par rapport aux prévisions) et imprévus ${ }^{12}$ (identification, gestion, explication et sentiments éprouvés).

Nous avons utilisé le logiciel $\operatorname{ALCESTE}^{13}$, pour une exploration multidimensionnelle des questions abordées. Ce logiciel pratique sur les données une classification descendante hiérarchique (CDH) puis une analyse factorielle des correspondances (AFC). La CDH associe aux classes des modalités différentes des variables, construisant ainsi des profils-types de sujets épistémiques, l'AFC fait apparaître sur les plans factoriels l'opposition qui existe entre les modalités, mettant de la sorte à jour les principes organisateurs des prises de position par rapport aux repères communs.

Nous pensons, avec A. PIASER (1998), que la prise en compte des différences d'association entre modalités à l'intérieur d'une même classe ou sur un même facteur permet, à travers la lecture des profils de classe et de la constitution des facteurs, l'approche des représentations des sujets, car ce sont les réponses fournies par les enseignants au questionnaire, qui dictent les possibilités d'association entre ces modalités.

\section{Les résultats}

Nous nous proposons d'évoquer les résultats issus de la CDH (classes de réponses) et de l'AFC (ancrage des prises de position par rapport aux principes organisateurs de la représentation), puis de présenter un modèle d'intelligibilité, outil de lecture des pratiques déclarées, que ces résultats ont permis de construire.

\section{Cinq classes de réponses ( $\mathrm{CDH})$}

Ce corpus est tout d'abord divisé en deux grandes classes qui apportent une première distinction générale éclairant les partitions ultérieures.

Qu'il s'agisse en effet de la gestion des imprévus ou de l'idéal professionnel, elles ébauchent, dans les grandes lignes, les modèles du pédagogue et du magister décrits par M. HIRSCHHORN (1993).

La classe 8 des "pédagogues" apparaît habituée aux situations proposées qui ne sont pas ou plus des imprévus et qu'elle gère en s'y adaptant (improvisation, construction de notion), ou en se centrant sur le contrat pédagogique défini selon P. PERRENOUD (1996).

Elle rassemble des enseignants qui se décrivent dans des rapports de valeur, mais aussi d'écoute avec leurs élèves (Soi professionnel), considèrent nécessaire le travail de construction de cours par l'enseignant, participent au travail en équipe au niveau des disciplines, et souhaitent améliorer leurs pratiques par la pédagogie différenciée (idéal professionnel).

Pour la classe 7 des " magisters", les situations proposées sont au contraire des imprévus, qu'elle gère en se centrant sur les prévisions, quitte à exclure des élèves de cours en cas de difficultés relationnelles.

Les professeurs qui relèvent de cette classe entretiennent un rapport affectif à leur discipline (idéal professionnel) et ne participent pas à la formation continue.

\footnotetext{
${ }^{12}$ Comme le dysfonctionnement, la contestation, les digressions, le travail non fait, les oublis de matériel, les tâches administratives, les aléas de la salle ...

${ }^{13}$ Pour une présentation complète, voir M. REINERT (1990).
} 
La classe $1^{14}$, “le pédagogue distancié", à travers les réponses qu'elle rassemble, brosse le profil d'un enseignant attaché à la conduite pédagogique de la classe et prêt à assumer les problèmes relationnels qui se posent.

Les pratiques déclarées apparaissent ainsi en accord avec la représentation de pédagogue que l'enseignant a de lui-même (Soi professionnel) et en cohérence avec son idéal professionnel (pédagogie différenciée, par exemple). Son implication psychosociale, produite par un engagement associatif, semble lui permettre de garder une certaine distance affective. Si l'affectivité n'est pas absente, en particulier de l'idéal professionnel, elle semble maîtrisée, apprivoisée, dans l'espace des pratiques professionnelles.

La classe 2, du "pédagogue libertaire ", témoigne de l'intérêt pour les aspects éducatifs et relationnels de la profession, est liée à une implication psychosociale — générée par un engagement associatif, même modéré-, et une image de Soi attachée à des valeurs, associées à un contexte d'exercice difficile (ZEP).

L'ancienneté professionnelle et dans le collège permet de prendre des libertés avec l'institution (programme, horaires).

"Pédagogue libertaire " donc, en référence à la visée de développement et d'affirmation de la personnalité de chaque individu, quitte à ne pas respecter les contraintes scolaires (HIRSCHHORN, 1993).

Dans la classe 3, "le modèle métissé ", évoque à la fois le pédagogue et le magister : pédagogue par la place du contrat pédagogique et son adaptation aux difficultés des élèves, mais magister aussi par la valeur centrale que constitue le savoir, inférée à travers l'importance accordée à la discipline.

La classe 4, celle du "magister externe", regroupe des réponses d'enseignants qui maintiennent les prévisions et tendent à expliquer les imprévus par des causes externes ou par le contexte. Par exemple l'enseignant gère l'excitation de la classe en recentrant sur le travail, quitte à exclure un élève de cours. Le travail non fait par les élèves s'explique, pour lui, par leur manque d'envie de travailler, et le dysfonctionnement de la classe par leur absence d'autonomie ou la position de l'heure dans la journée.

La classe $5 \mathrm{du}$ "magister interne" rassemble des réponses qui se centrent sur la discipline : désir de faire partager son intérêt, report éventuel de la fin du cours en réduisant aux objectifs minima, culpabilisation si le contenu est différent. Ceci semble favoriser l'émergence d'imprévus — dont l'enseignant a tendance à se sentir responsable- face à un public dont il a du mal à évaluer les difficultés.

Voici la CDH schématisée :

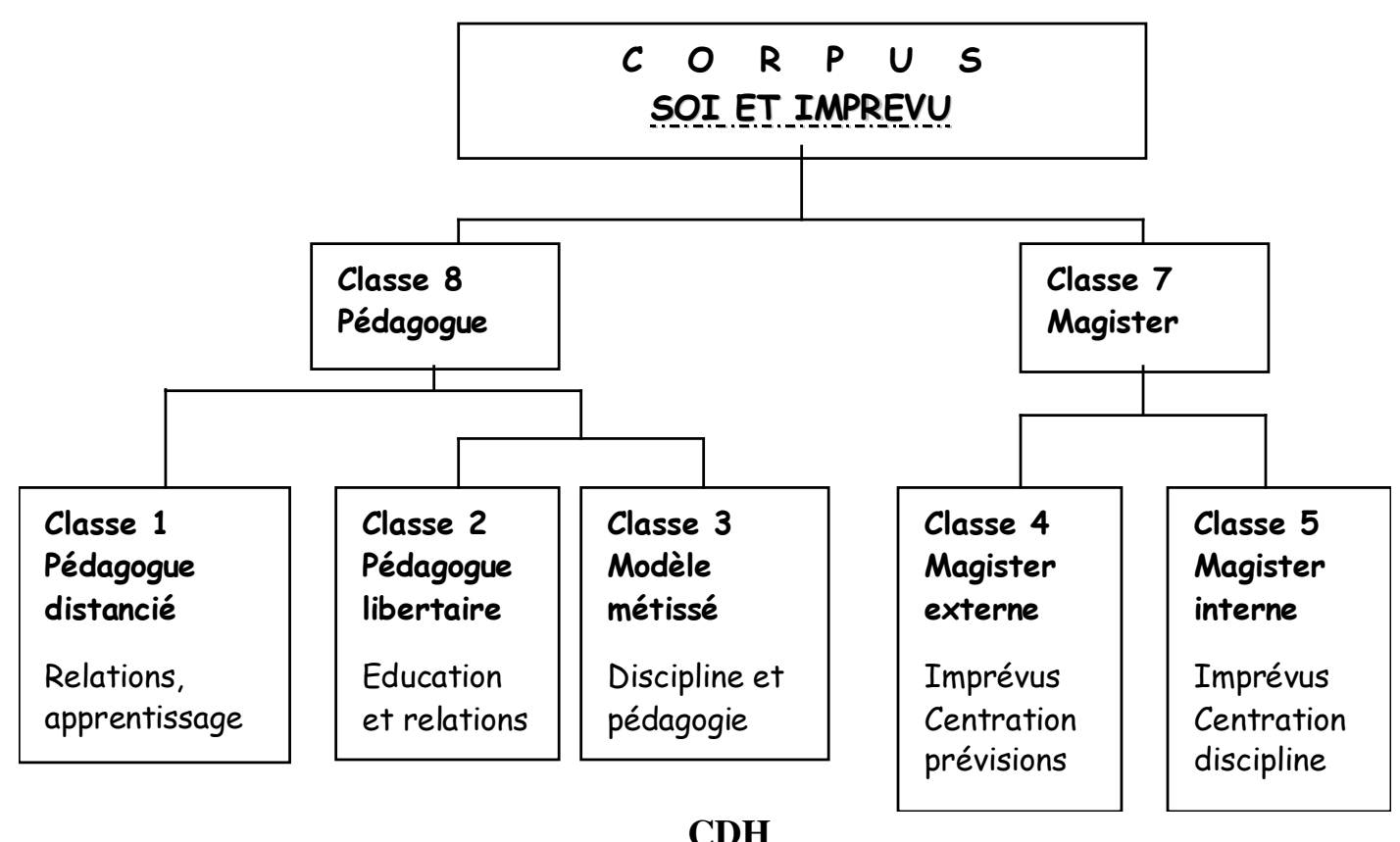

Trois principes organisateurs de la représentation (AFC)

Quatre facteurs ont été repérés, nous n'en présenterons que trois. Les deux premiers facteurs traitent d" "identification et gestion des imprévus" et du " sentiment de contrôle".

${ }^{14} 36$ uce soit $24,83 \%$ / X2 moyen : 7.30 . 
Nous insérons ici le premier plan factoriel (facteurs croisés 1 et 2 ; $\mathrm{C} 1, \mathrm{C} 2$ pour classe 1, classe 2...), de façon à en éclairer la description :

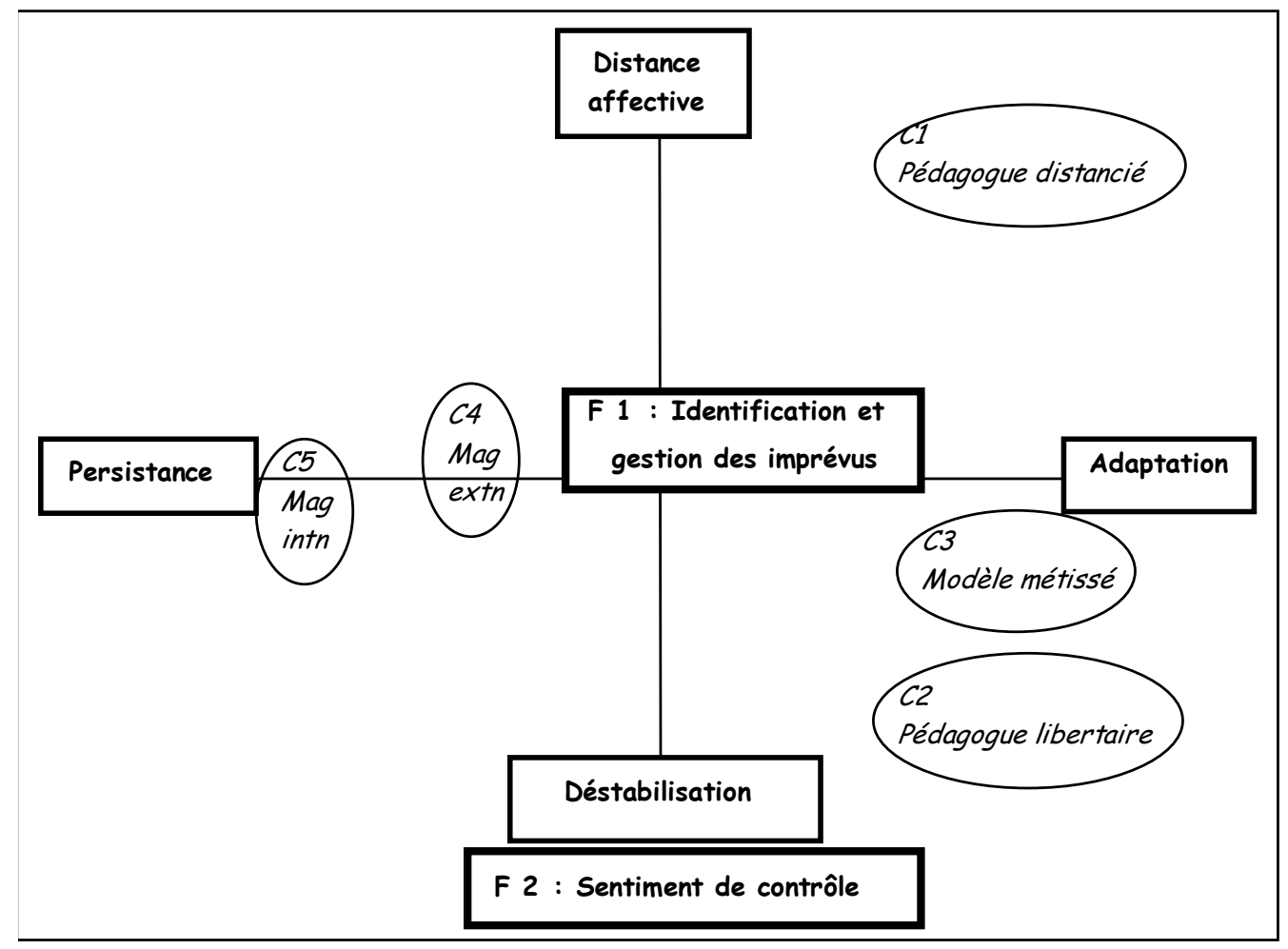

Le premier facteur ${ }^{15}$, “identification et gestion des imprévus", s'étire du pôle "adaptation" au pôle "persistance ", distinguant par là les deux modèles d'enseignants.

- Au pôle " adaptation ", les classes des " pédagogues", avec les situations proposées (par le questionnaire) non identifiées comme des imprévus. L'enseignant s'adapte en improvisant, y compris par rapport aux prévisions. Il considère que c'est dans l'ordre des choses et s'appuie ainsi sur les émergences du contexte.

Là se situent des enseignants engagés professionnellement (mouvements pédagogiques, syndicats, qualité de professeur principal, travail en équipe), avec une ancienneté professionnelle relativement importante (11-20 ans, voire 31-42 ans), qui ont l'habitude de s'adapter aux imprévus.

- Au pôle " persistance", les classes des " magisters", avec les situations identifiées comme des imprévus. L'enseignant les explique par sa propre responsabilité (attribution interne) ou par celle de ses élèves (attribution externe) ${ }^{16}$. Quoi qu'il en soit, il maintient sa préparation (persistance) : lorsqu'il y a de nombreux absents, il maintient le contrôle; en cas de digression, il recentre.

Il s'agit d'enseignants nouveaux dans la profession et/ou dans le collège ${ }^{17}$, qui ne sont pas très engagés professionnellement, et persistent dans leurs prévisions malgré les imprévus.

Le deuxième facteur ${ }^{18}$, "sentiment de contrôle", différencie le "pédagogue distancié " au pôle "distance affective ", du "pédagogue libertaire" au pôle " déstabilisation".

- Au pôle " distance affective ", le " pédagogue distancié " (Classe 1) éprouve le sentiment de contrôler la situation et se place au plan professionnel : face aux imprévus didactiques, il éprouve une satisfaction professionnelle ou un sentiment de perte de temps ; et face à ceux touchant aux aspects relationnels, il est concerné seulement professionnellement, prêt à les traiter, cela fait partie de son travail.

\footnotetext{
${ }^{15} \mathrm{VP}: .0396 / 39,49 \%$ de l'inertie.

${ }^{16}$ Ainsi, n'aimant pas les tâches administratives, il les oublie ; la notion à acquérir est parfois trop difficile pour les élèves parce qu'il a du mal à en évaluer la difficulté ; mais si la classe “dysfonctionne " (agitation, leaders...), c'est dû à des élèves nerveux ou caractériels.

17 1-5 ans d'ancienneté professionnelle et première année dans le collège.

${ }^{18} \mathrm{VP}$ : .0235 / 23,52\% de l'inertie.
} 
De plus il se centre sur le groupe-classe pour des questions relationnelles -il cherche à comprendre ce qui se passe—, ou cognitives — découverte par les élèves de voies différentes dans la construction d'une notion.

Ce sont des enseignants nouveaux dans la profession, fortement engagés sur le plan politique et associatif (implication psychosociale), qui ont le sentiment de contrôler la situation.

- A l'autre pôle, "déstabilisation ", le "pédagogue libertaire" (Classe 2) et même le " modèle métissé " (Classe 3) ressentent affectivement les imprévus didactiques et relationnels. Ils sont culpabilisés par les imprévus didactiques, et touchés personnellement et souvent dépassés par les imprévus relationnels.

Ce sentiment de ne pas bien contrôler la situation est là aussi lié à certaines caractéristiques de gestion des imprévus, et de sens donné aux situations. L'enseignant se centre sur ses prévisions, semble attaché au contrat pédagogique. Il prend une heure de plus pour pallier la lenteur de certains élèves et gère une éventuelle excitation de la classe par des régulations, voire des exclusions du cours.

Il s'agit d'enseignants anciens dans la profession et le collège, qui témoignent d'une implication psychosociale modérée (engagement modéré dans des mouvements associatifs), et sont déstabilisés par les imprévus.

Notons que, dans les réponses de chacun, se repèrent sans doute des effets de contexte.

La zone du collège relève en effet à la fois des facteurs 1 et 2 :

- ZEP aux pôles " adaptation " (F1) et " déstabilisation " (F2),

- collège sans classement particulier aux pôles " persistance " (F1) et " distance affective " (F2).

Les ressources et contraintes des contextes n'étant pas les mêmes, l'identification et la gestion des imprévus (F1), et le sentiment de contrôle (F2) apparaissent dès lors comme des principes organisateurs de représentations contextualisées.

Voici à présent le plan factoriel présentant le facteur 1 croisé avec le facteur 3 :

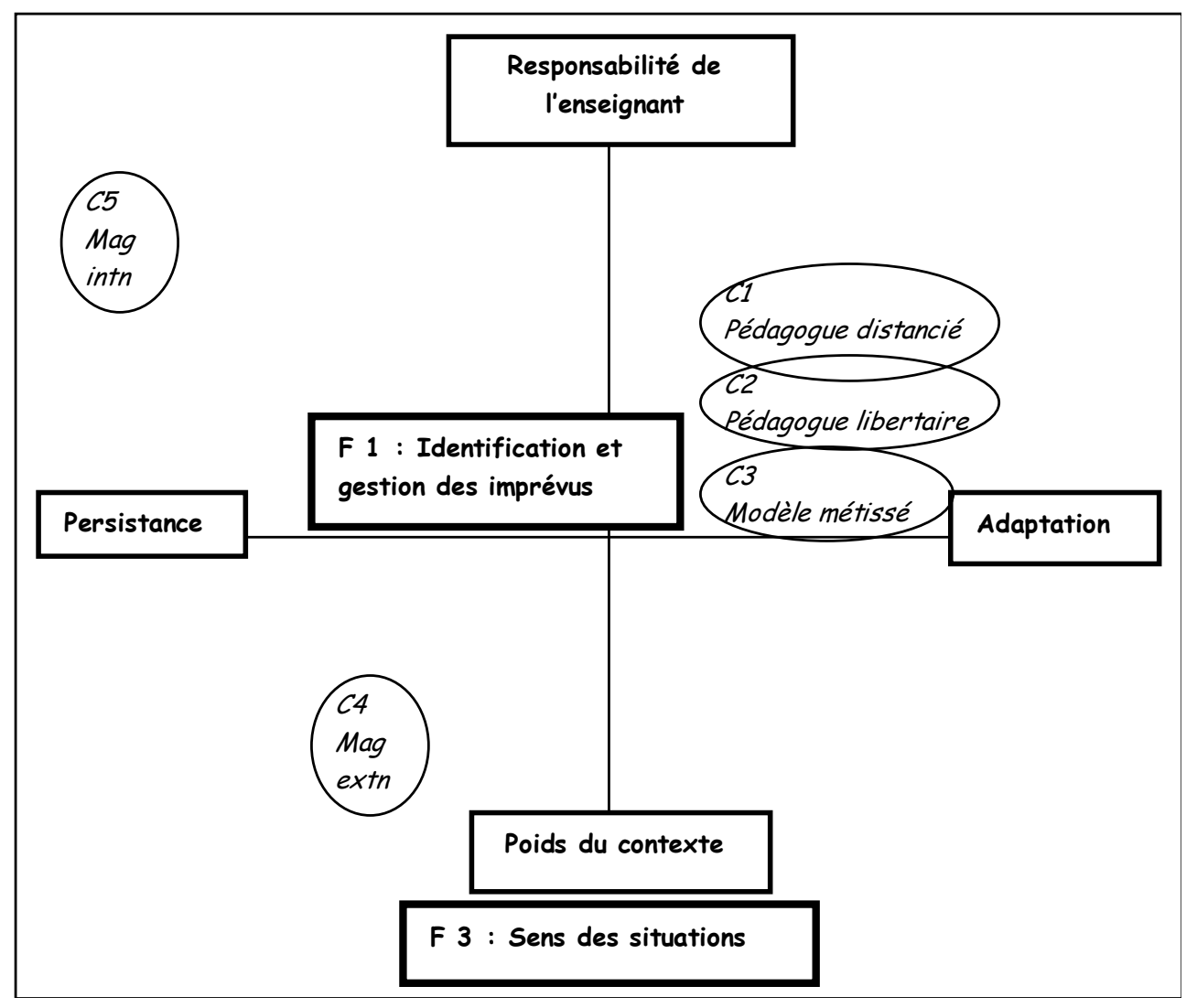

Le troisième facteur ${ }^{19}$ introduit une différence dans la classe des magisters en mettant l'accent sur le "sens des situations ", avec le pôle "responsabilité de l'enseignant" au Nord (Classe 5 " magister interne "), et le pôle " poids du contexte" au Sud (Classe 4 " magister externe") :

\footnotetext{
${ }^{19} \mathrm{VP}$ : .0189 / 18,89\% de l'inertie.
} 
- Au Nord, l'enseignant se réfère à l'interaction pédagogique pour expliquer le dysfonctionnement de la classe (désintérêt par incompréhension), et à des causes internes pour expliquer les digressions (faire partager son intérêt).

Il s'agit là de professeurs encore "nouveaux" dans le collège (2-5 ans), en milieu rural, et qui se sentent responsables de ces situations.

- Au Sud, pour expliquer le dysfonctionnement de la classe, il se réfère au contexte (position de l'heure dans la journée scolaire), et se centre sur ses prévisions avec recours à la mobilisation des élèves pour gérer l'excitation de la classe.

Ce sont des professeurs anciens dans la profession et le collège, en milieu urbain, très impliqués professionnellement (formation continue) ; ils " relativisent" les situations imprévues, connaissent "le métier" et l'établissement.

\section{INTERPRETATIONS : AU CEUR DE L'IMPREVU}

L'ancienneté professionnelle et l'ancienneté dans le collège, différentielles des représentations Voici un tableau récapitulatif sur lequel s'appuie l'interprétation proposée ensuite. Rappelons qu'il s'agit toujours là de portraits de sujets épistémiques, inscrits donc dans des tendances.

\begin{tabular}{|l|l|l|l|}
\hline \multicolumn{2}{|c|}{$\begin{array}{c}\text { Professeurs anciens } \\
\text { (profession et collège) }\end{array}$} & \multicolumn{2}{c|}{$\begin{array}{c}\text { Professeurs nouveaux } \\
\text { (profession et collège) }\end{array}$} \\
\hline \multicolumn{1}{|c|}{ pôles } & \multicolumn{1}{c|}{ contexte / ancienneté } & \multicolumn{1}{c|}{ pôles } & \multicolumn{1}{c|}{ contexte / ancienneté } \\
\hline Adaptation & $\begin{array}{l}\text { ZEP / } \\
\text { ancien prof }\end{array}$ & Persistance & $\begin{array}{l}\text { Coll non ZEP / } \\
\text { nouveau prof + nouveau coll }\end{array}$ \\
\hline Déstabilisation & $\begin{array}{l}\text { ZEP / } \\
\text { ancien prof + ancien au coll }\end{array}$ & Distance affective & $\begin{array}{l}\text { Coll non ZEP / } \\
\text { nouveau prof + nouveau au coll }\end{array}$ \\
\hline Poids contexte & $\begin{array}{l}\text { urbain / } \\
\text { ancien prof + ancien au coll }\end{array}$ & $\begin{array}{l}\text { Responsabilité } \\
\text { enseignant }\end{array}$ & $\begin{array}{l}\text { rural / } \\
\text { nouveau au coll }\end{array}$ \\
\hline
\end{tabular}

\section{Contexte, ancienneté et imprévus}

Les variables de l'ancienneté professionnelle et dans le collège sont différentielles des représentations : la première, dans le domaine de l'identification et de la gestion des imprévus —avec l'ancienneté s'acquièrent des conduites adaptatives- ; toutes les deux dans le domaine du sentiment de contrôle et du sens donné aux situations.

Ainsi les professeurs nouveaux dans la profession et le collège, qui focalisent sur leurs prévisions face aux imprévus, maintiennent une distance affective, les nouveaux sur l'établissement se sentant responsables des imprévus. Les professeurs les plus anciens dans la profession et le collège, s'adaptent aux imprévus, sont parfois déstabilisés, et invoquent des effets de contexte.

\section{Des représentations contextualisées}

D'autre part le contexte comporte des contraintes dont la force plus ou moins grande module les réponses.

Les collèges sans classement particulier sont associés à la persistance et à la distance affective, tandis que les ZEP le sont à l'adaptation et à la déstabilisation. De même le milieu rural est associé au sentiment de responsabilité de l'enseignant face aux imprévus, tandis que le milieu urbain l'est au sentiment du poids du contexte.

Un contexte difficile déstabilise donc les enseignants, même s'ils sont anciens dans la profession, et cette déstabilisation est peut-être ce qui les pousse à s'adapter ${ }^{20}$, sans doute grâce à leur ancienneté —ils “ ont du métier".

Par contre un contexte sans difficulté particulière permet aux nouveaux professeurs (également nouveaux sur le collège) de s'en tenir à leurs prévisions et de maintenir une distance affective face aux imprévus.

Les enseignants apparaissent plus enclins à invoquer des effets de contexte en milieu urbain qu'en milieu rural — sans doute moins anonyme ${ }^{21}$ — où semble jouer le processus d'attribution interne.

\footnotetext{
${ }^{20}$ Il est également permis de penser que dans un contexte difficile, l'enseignant s'efforcera d'adapter son projet —et sans aucun doute l'expérience accumulée au fil des ans et des bouleversements du système éducatif l'y aidera—, alors que dans un contexte sans difficulté majeure il s'efforcera malgré tout de le réaliser —et l'on peut alors effectivement imaginer que l'expérience du novice ne lui laisse pas vraiment d'autre choix que “ d'appliquer " le projet qu'il a prévu.

${ }^{21}$ Les effectifs souvent plus modestes permettent aux acteurs de se connaître plus facilement, les enseignants ont peutêtre plus de contacts avec élèves et parents d'élèves — y compris hors de l'établissement—, les gens se connaissent plus
} 
Nous constatons donc que les représentations sont contextualisées, ici dépendantes du contexte dans lequel sont socialement insérés les sujets.

Toutefois l'enseignant n'est jamais entièrement déterminé ni entièrement souverain, et d'autres variables interviennent dans l'approche de l'imprévu.

\section{Interactions contexte - ancienneté - implication : un outil de lecture}

Nous allons présenter un schéma synthétique en forme d'étoile, de façon à mettre en évidence les divers éléments en interaction ${ }^{22}$.

Sont inscrits à l'intérieur de l'étoile les axes des principes organisateurs des représentations professionnelles de la gestion des imprévus, avec leurs pôles auxquels se rattachent les modèles de référence. Les voici rappelés dans le tableau suivant :

\begin{tabular}{|c|c|}
\hline AXES & POLES \\
\hline Facteur 1 : & "adaptation - persistance " \\
\hline Identification et gestion des imprévus & pédagogue \\
\hline Facteur 2 : & "distance affective - déstabilisation " \\
pédagogues \\
\hline Fentiment de contrôle & "responsabilité de l'enseignant - poids du contexte" \\
Sens des situations & magisters \\
\hline
\end{tabular}

\section{$\underline{\text { Axes de l'AFC et modèles normatifs }}$}

Nous envisageons chacun de ces axes comme un continuum aux extrémités polarisées (W.DOISE, 1992), sur lequel les enseignants se déplaceraient en fonction du contexte, de leur ancienneté et de leurs implications, et sans doute de bien d'autres éléments encore.

Toutefois dans une perspective de réduction de la complexité, nous indiquons les variables illustratives les plus discriminantes pour les sujets épistémiques de notre échantillon. Ces variables, placées aux pointes de l'étoile sont l'ancienneté (profession / collège) traitée avec le contexte, et l'implication (professionnelle / psychosociale).

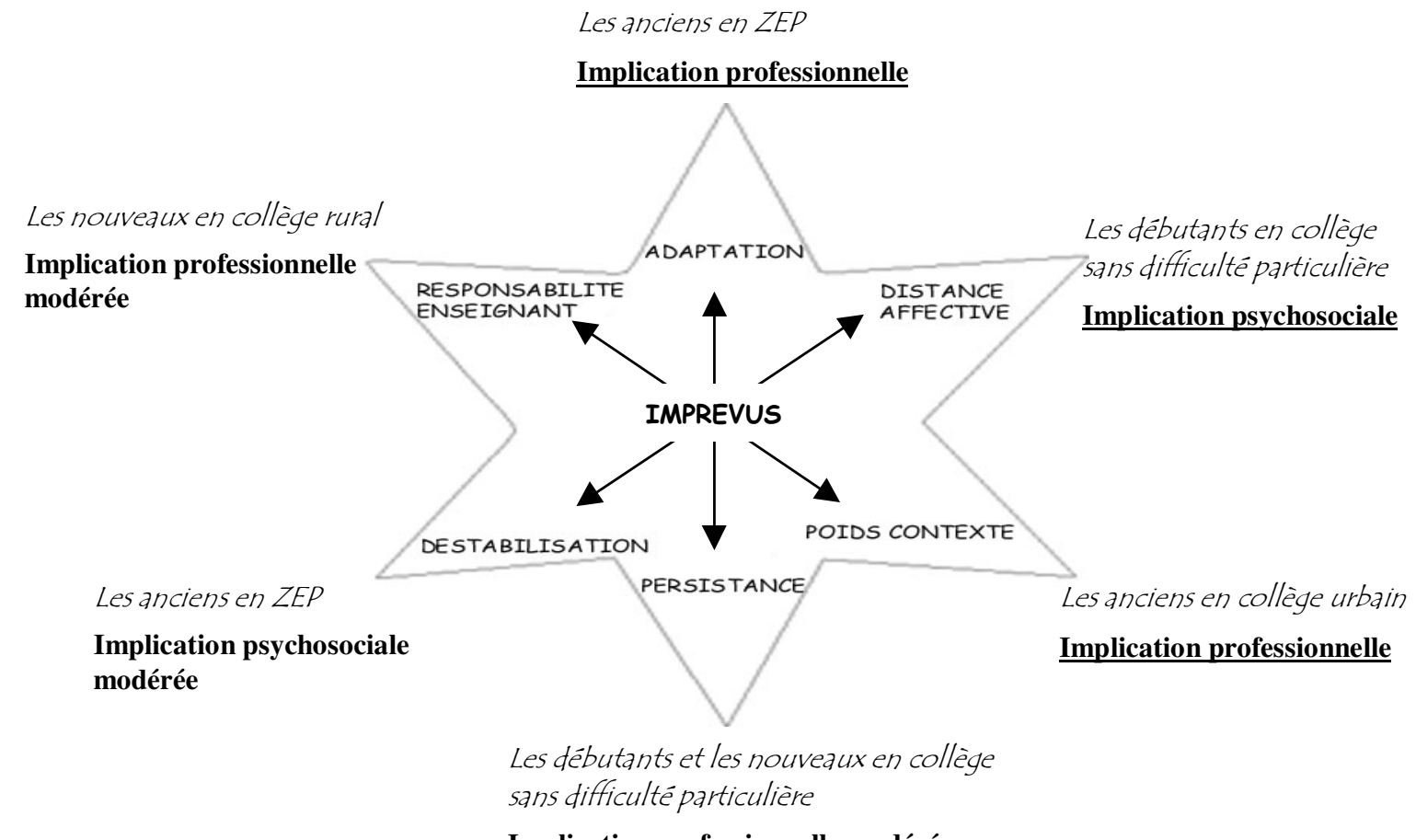

$\underline{\text { Modèle d'intelligibilité }}$

facilement et peuvent avoir une vie sociale plus fréquente; et même ceux qui ne vivent pas sur le lieu de travail, ne peuvent ignorer le hors-champ de la classe, ne serait-ce que par les contacts avec les collègues.

${ }^{22}$ Lorsque les modalités de l'ancienneté dans la profession et dans le collège s'opposent terme à terme aux pôles du même axe, priorité est donnée à l'ancienneté dans la profession (“ anciens " / " débutants ") ; mais si un axe oppose les modalités de l'ancienneté dans la profession et que celles de l'ancienneté dans le collège apparaissent à un seul pôle, la nuance, significative, est précisée (“ anciens " / " nouveaux ”). 
On constate que l'implication professionnelle ou psychosociale s'oppose sur chaque axe factoriel dans ses modalités "forte" et "modérée", et que, hormis sur le facteur du sentiment de contrôle, les fortes implications sont associées à des contextes difficiles et aux enseignants anciens.

Ce qui autoriserait à penser que c'est dans la difficulté au quotidien et avec l'expérience que se renforcent les engagements et, donc, les implications.

Tout d'abord l'implication professionnelle :

Ainsi dans un contexte difficile, une forte implication professionnelle peut aider à prendre de la distance pour gérer les imprévus ou les expliquer. D'autant plus lorsqu'elle s'appuie sur l'expérience professionnelle et la connaissance du lieu d'exercice que confère l'ancienneté. On pourrait également penser qu'elle a été rendue nécessaire, au fil des ans, par la complexité du contexte et des relations qui s'y tissent. Le passé de réformes et le vécu des bouleversements consécutifs ont pu entraîner une évolution des représentations, contrairement aux enseignants nouveaux dans la profession, qui de plus ne témoignent pas d'une implication professionnelle forte. Les premiers appartiennent, dans la $\mathrm{CDH}$, aux classes des pédagogues, les seconds à celles des magisters.

Ensuite l'implication psychosociale :

L'implication psychosociale apparaît associée à des aspects plus affectifs, qu'il s'agisse, pour la modalité forte, de distance affective chez les nouveaux, ou, pour la modalité modérée, de déstabilisation chez les anciens.

Des différences de perception et de gestion des imprévus sont donc bien liées à l'implication professionnelle et psychosociale: l'implication résultant d'engagements professionnels ou psychosociaux, associée au contexte et à l'ancienneté, permet adaptation et distance affective. Paradoxalement, plus l'enseignant est impliqué, plus il peut prendre du recul par rapport aux événements et s'y adapter, aidé en cela généralement par son ancienneté. Par contre avec une implication plus faible, il persiste dans ses prévisions, il est déstabilisé et se sent responsable des situations imprévues. Il s'agit souvent là de professeurs nouveaux dans le collège.

Où l'on retrouve la problématique du triptyque agent, acteur, auteur (J. ARDOINO, 2000).

Ainsi les débutants de l'échantillon, qui témoignent d'une implication professionnelle modérée, sont plus ou moins malmenés par les imprévus. Ils se raccrochent à leur préparation, apparaissant en cela agents. Mais ces débutants peuvent aussi disposer d'initiatives propres qui leur permettent d'élaborer du sens dans leur conduite de classe face aux imprévus, ils deviennent alors acteurs, construisant ainsi leur expérience, voire auteurs lorsqu'ils inventent des réponses appropriées.

Au contraire, les anciens professeurs qui connaissent une forte implication professionnelle, peuvent utiliser leurs ressources pour s'adapter aux imprévus, quitte à inventer leurs propres réponses, en auteurs qu'ils ont pu devenir avec le temps.

Pour construire ce "modèle", nous avons souligné certains aspects de la complexité de conduite face aux imprévus. Cette représentation schématique ne correspond donc pas strictement à la réalité : les enseignants se déplaceraient plutôt sur chaque continuum en fonction des circonstances, de leur histoire et de leur implication. C'est pourquoi l'on peut penser qu'un sujet réel est tour à tour, et tout au long de sa vie professionnelle, agent, acteur, auteur. Les conduites témoignent par là d'une variabilité importante. Aussi allons-nous utiliser ce modèle d'intelligibilité pour relire les entretiens de la première étape de cette recherche.

\section{Une relecture d'entretien à l'aide du modèle présenté}

Nous pouvons ainsi retrouver chez un même enseignant des éléments relevant de chaque continuum. L'analyse thématique d'un entretien a mis à jour chez un même professeur, à travers des éléments de son histoire personnelle, un changement de modèle de référence : d'ancien pédagogue, il est devenu magister, et ses discours en portent la trace. Nous proposons ici, à titre d'exemple, des extraits de son entretien pour lesquels nous indiquerons entre parenthèses les pôles auxquels ils se rattachent.

\section{Axe de l'identification et de la gestion des imprévus.}

- "Il me semble même que les premiers cours, je les ai faits avec la feuille à la main, quasiment. Mais maintenant, non, je ne m'en rends même pas compte. C'est vrai qu'on s'adapte en permanence, si on sait que dans la classe il y a un travail de recherche ou de discussion, fatalement on rebondit sur la réponse, on ne perd pas en gros le cap, on sait bien à peu près où on va aller mais, pour y aller, on peut prendre des voies différentes" (pôle " adaptation", pédagogue). 
- "Si je suis pressé, fatalement je vais me limiter à ceux qui répondent le mieux, qui se coulent dans le moule" (pôle "persistance", magister).

Axe du sentiment de contrôle.

- "Ca ne me pose plus de problème, franchement, ce que je n'ai pas fait là, je le fais le lendemain. Quand ça arrive, non, c'est dans l'ordre des choses " (pôle "distance affective ", pédagogue)

- “Je m'adapte, il me semble, assez mal, malgré tout, à l'hétérogénéité des classes, qui me paraît de plus en plus importante et pour laquelle je n'ai pas de recette, je ne sais pas quoi faire" (pôle "déstabilisation ", pédagogue).

Axe du sens des situations.

- "Dans ma classe, je trouve que (les plus faibles) sont souvent laissés de côté et que par ailleurs je ne donne pas non plus aux meilleurs ce qu'ils pourraient attendre. Je n'exige pas d'eux ce qu'on pourrait exiger. Donc là, c'est une source permanente d'insatisfaction. C'est un problème que je n'ai pas résolu, et puis je ne vois pas comment le résoudre" (pôle " responsabilité de l'enseignant ", magister).

- "Maintenant on a des classes de plus en plus hétérogènes. Le passage se fait automatiquement, selon les voux des familles. L'ambiance, de ce point de vue là. Moi, j'ai en quatrième des élèves qui auraient dû aller même en apprentissage. Ils n'ont pas trouvé d'apprentissage, donc ils sont en quatrième. Ils savent à peine lire et écrire, qu'est-ce que je fais, moi " (pôle “ poids du contexte ", magister).

Se référant tour à tour aux modèles normatifs du pédagogue ou du magister, les discours de cet ancien enseignant témoignent bien, nous semble-t-il, de la variabilité des représentations, et de ce déplacement sur chaque facteur conçu comme un continuum.

\section{CONCLUSION}

Ainsi la façon dont les professeurs gèrent les situations imprévues diffère selon leur modèle sous-jacent de référence, “ modèles normatifs" qui n'existent pas à l'état “pur” dans la réalité (M. HIRSCHHORN, 1993). Ils sont " métissés ", " altérés ", et les enseignants, suivant les circonstances, forcent le trait d'un modèle ou de l'autre.

Les divergences repérées parmi les enseignants qui se réfèrent au même modèle, sont liées à leur histoire personnelle, aux contextes professionnels dans lesquels ils sont insérés, et à leurs diverses implications.

Et si le contexte est déterminant, l'ancienneté (dans ses deux dimensions) et les implications (professionnelles et psychosociales) sont différentielles des représentations: l'enseignant est bien “contextualisé / contextualisant" (M. BRU, 1994).

Il ressort ainsi que, plus l'enseignant est impliqué,

- plus il a de distance par rapport aux imprévus — toutefois dans un contexte sans difficulté particulière,

- plus il peut se détacher de sa préparation pour s'adapter aux situations dans un contexte souvent très difficile — mais il a l'expérience conférée par l'ancienneté,

- plus il peut marquer de distance dans l'attribution de sens aux situations, même dans un contexte relativement difficile - aidé en cela par sa forte implication professionnelle et son ancienneté dans le collège.

On le voit, la professionnalité enseignante se construit bien pour partie dans le "faire avec" les imprévus, et l'implication joue ici un rôle majeur.

Dès lors la question pourrait être : comment accroître l'implication des enseignants pour que se construise leur professionnalité dans la confrontation aux imprévus? Les échanges entre enseignants pourraient constituer une réponse, pour apprendre de l'expérience des autres, se former à traiter l'imprévu (P. PERRENOUD, 1999), dans une perspective de construction des représentations des imprévus et des possibilités d'adaptation. Et toute pratique étant contextualisée, donc difficilement reproductible à l'identique, la "pédagogie comme récit d'éducation" (P. MEIRIEU, 1997) a l'avantage de présenter un caractère formateur. Le récit, avec les échanges qu'il entraîne, permet une décentration par le déplacement sur le point de vue de l'autre, décentration susceptible de transformer les représentations. Il s'agirait en quelque sorte de se laisser "altérer" par ces récits, pour ensuite les altérer soi-même, de façon à inventer ses propres réponses, et devenir ainsi auteur de ses actions. 
Les récits dont parle P. MEIRIEU nous semblent en effet bien plus que de simples échanges d'expériences. Ces récits du "faire avec" nous paraissent à même de susciter et de nourrir une implication apparue si importante dans la confrontation à l'imprévu.

\section{REFERENCES BIBLIOGRAPHIQUES CITEES}

ARDOINO, J. (2000). Les avatars de l'éducation. Paris : PUF.

BATAILLE, M. et al. (1997). Représentations sociales, représentations professionnelles, système des activités professionnelles. L'année de la recherche en sciences de l'éducation, 1997, 57-89.

BATAILLE, M. (2000). Représentation, implicitation, implication : des représentations sociales aux représentations professionnelles. In : GARNIER, C., ROUQUETTE, M-L. (éd.). 2000. Représentations sociales et éducation. Paris : Editions Nouvelles, 165-189.

BENAÏOUN-RAMIREZ, N. (1999). L'imprévu dans les conduites de classe, "faire avec". Communication au $3^{\text {ème }}$ congrès international de l'Actualité de la Recherche en Education et Formation (AECSE), 28, 29, 30 juin 1999.

BENAÏOUN-RAMIREZ, N. (2001). Contribution à l'étude de la professionnalité enseignante: des enseignants de collège face à l'imprévu en classe. Thèse de doctorat en Sciences de l'Education. Direction M. BATAILLE. Toulouse, UTM.

BRU, M. (1993). L'enseignant, organisateur des conditions d'apprentissage. In : J. HOUSSAYE, La pédagogie: une encyclopédie pour aujourd'hui, 103-117.

BRU, M. (1994). Quelles orientations pour les recherches sur la pratique de l'enseignement ? L'Année de la recherche en sciences de l'éducation, 1994, 165-174.

COSTALAT-FOUNEAU, A-M. (1997). Identité sociale et dynamique représentationnelle.

DOISE, W., CLEMENCE, A., LORENZI-CIOLDI, F. (1992). Représentations sociales et analyses de données. Grenoble : PUG.

HENRIQUEZ, S. (1989). Changements opérés chez les enseignants : continuité et ruptures des représentations et des pratiques enseignantes, Education comparée, 42, 1989, 99-112.

HIRSCHHORN, M. (1993). L'ère des enseignants. Paris : PUF.

JACQUET-MIAS, C. (1998). L'implication professionnelle des travailleurs sociaux dans le secteur médico-social associatif. Paris : L'Harmattan.

LAHIRE, B. (1998). L'homme pluriel, les ressorts de l'action. Paris : Nathan.

MEIRIEU, P. (1997). Praxis pédagogique et pensée de la pédagogie. Revue Française de Pédagogie, INRP, juillet-aoûtseptembre 1997, 120, 25-37.

PERRENOUD, P. (1996). Enseigner, agir dans l'urgence, décider l'incertitude. Paris : ESF éditeur.

PERRENOUD, P. (1999). Gestion de l'imprévu, analyse de l'action et construction de compétences. Education Permanente, 1999, 140, 3, 123-144.

PIASER, A. (1998). Représentations professionnelles à l'école. Particularités selon le statut : enseignant, inspecteur. Thèse de doctorat en Sciences de l'Education. Direction M. BATAILLE. Toulouse, UTM.

REINERT, M. (1990). Une méthodologie d'analyse des données textuelles et une application : Aurélia de G. de Nerval. Bulletin de méthodologie sociologique, 26, march 1990, 25-53.

ROUQUETTE, M-L (1997). La chasse à l'immigré. Violence, mémoire et représentations. Liège : Mardaga éditeur.

TAP, P., ESPARBE-PISTRE, E., SORDES-ADER, F. (1997). Identité et stratégies de personnalisation. Bulletin de psychologie, janvier-avril 1997, L, 428, 185-196.

TOCHON, F.V. (1993). L'enseignant expert. Paris : Nathan.

WOODS, P. (1990). L'Ethnographie de l'Ecole. Paris : Armand Colin.

Circulaire (23/5/97) : Mission des professeurs exerçant en collège, lycée d'enseignement général et technologique ou en lycée professionnel. B.O. n²2 du 29 mai 1997. 